\title{
Case Report: Coronavirus Disease-19 Pandemic Associated Gaming Disorder
}

\author{
Syaifuddin Nasution ${ }^{1}$ (D), Mustafa M. Amin ${ }^{2 *}$ (D) Elmeida Effendy ${ }^{2}$ (D) \\ ${ }^{1}$ Department of Psychiatry, Faculty of Medicine, Universitas Sumatera Utara, Medan, Indonesia; ${ }^{2}$ Department of Psychiatry, \\ Faculty of Medicine, Universitas Sumatera Utara, Medan, Indonesia
}

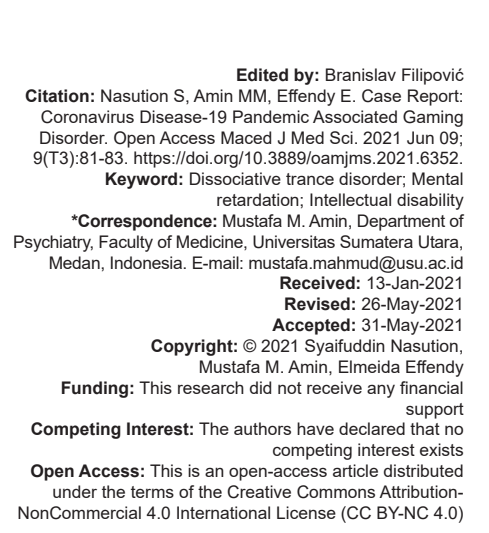

Abstract

BACKGROUND: Self-quarantine and social distancing are one of the numerous impacts of coronavirus disease (COVID-19) pandemic that leads individuals to spend much more time at home which in turn interestingly contributes to the increased number of gaming disorder cases. Gaming disorder is defined as a negative behavioral pattern that is characterized by excessive time spent on playing games and losing self-control in terms of playing games that causes neglect of other daily activities or interests.

CASE REPORT: We are reporting a case of gaming disorder in a 25-year-old man, D, through this COVID-19 pandemic. We suggest that there is an association between psychosocial stressors and environment with psychiatric conditions.

CONCLUSION: Further study is recommended to more holistically understand this particular gaming disorder.

\section{Introduction}

Coronavirus disease (COVID-19) cases were first reported in Wuhan in late 2019 and spread rapidly worldwide in a matter of months that lead to selfquarantine and social distancing. A study from China investigated that covid-19 is linked to depression that accounts for $17-48 \%$ of the population and $23-28 \%$ was found to experience anxiety disorder from which $4 \%$ appeared to be from university students population [1]. COVID-19 causes numerous changes in everyday activities, causing us to spend much more time at home that in turn leading to increased needs of entertainment, including those from online sources [2]. Online gaming has become much more prevalent, in the USA alone, it was reported that there was a significant increase of online gaming for as much as $75 \%$ at the beginning of March 2020 when the USA started to oblige protocols to prevent further spread of COVID-19 [3]. A similar thing also happens in India, in which people can even spend the whole day playing game, peak time at $8 \mathrm{pm}$ till midnight as if online gaming had become a part of social distancing itself [4].

Gaming disorder is defined as a negative behavioral pattern that is characterized by excessive time spent for playing game and losing self-control in terms of playing game that causes neglect of other daily activities or interests [5]. In accordance with DSM and ICD-11, gaming disorder is defined as repetitive behavior of playing game that continuously persists for at least 12 months [6]. In the Republic of Korea, internet/smartphone addiction prevalence has been increasing rapidly from $13.1 \%$ to $21.8 \%$ in $2013-2018$ (high risk: $1.3-2.7 \%$, mild risk 11.8-19.1\%). This has posed another social issue recently. Based upon age group, $29.3 \%$ of teenagers, $20.7 \%$ of kids, and $18.1 \%$ of adults are found to experience internet/smartphone addiction. Another study also revealed that among teenagers of $15-19$ years old, $8.4 \%$ male and $4.5 \%$ female were found to suffer from internet/smartphone addiction [6], [7].

Risk factors that contribute to gaming disorder are the following; biological, personality factor, psychological vulnerability, environmental factor, stress, and structure factors [8]. Gaming disorder is manifested as loss of self-control in terms of playing game that leads to other interests or priorities being abandoned, instead of acknowledging the negative consequence that may result from it. There are also several other disorders related to internet gaming disorder (IGD), including anxiety disorder, depression, suicidal thought, behavioral 
disorders, social phobia, autism spectrum disorder, attention deficit hyperactivity disorder, obsessivecompulsive disorder, and personality disorder [9].

\section{Case Report}

A 25-year-old man, D, is a university student from one of local university in North Sumatera, who came to our outpatient psychiatric clinic accompanied by his mother, complaining of a temper tantrum. According to his mother, D was also often seen to be very sad and anxious. These symptoms have been going on for 6 months and getting worse within the last month. His mother also admitted that $D$ is fond of playing game, either from his phone, personal computer, or from microconsole. D stated that he usually loves playing Mobile legend, PUBG mobile, free fire, Dota, Point blank, and Assassin Creed. He admitted that he started playing game for 9 years earlier with approximately $6-7 \mathrm{~h}$ spent for it on each day. Through this COVID-19 pandemic, he spent even more time playing game for as much as 10-12 h a day. He also stated that he has even much more leisure time at home recently, particularly because he does not have to go to university as all study activities are now limited to be from home. This turns into another consequence as D failed to cope up with his final assignment once again, after 3 years of postpone. $\mathrm{D}$ was always getting vigorously angry whenever being reminded or asked by his parents, denied of spending too much time playing game, and even spending much more time playing whenever he is in trouble. He only had very few friends at the campus but had many online friends, mostly those he met through online game. He had tried to cut his playing time, but he ended up giving up, as he no longer engaged to any hobby, even soccer, that he used to love playing before. This recent 1 month, $\mathrm{D}$ has always been anxious after long hours of playing the game, has difficulty of sleeping; sometimes he cannot even sleep at all, experiences temper tantrum, and has difficulty of concentrating.

D's parents are typically working parents who do not spend much time at home. Most of the time D was all by himself at home and playing game is seen as a way to kill time and loneliness. D just moved from another town and eventually has not been friends with anyone in his neighborhood. This is also one particular reason why he prefers playing game to involve in any other activities outside.

During interview session, our patient stated that he barely even sleeps at night, feels anxious and angry, as well as has difficulty of concentrating throughout the last 6 months and also noted that the symptoms were aggravated in the recent 1 month. Particularly during this pandemic, D admitted to becoming bad-tempered and even more anxious. We found that none of his family ever had any history of experiencing the same symptoms. General physical examination, neurological status, and his vitals were all measured to be within normal limits. Based upon psychiatric interview, we observed that generally, D is still very well groomed with normal psychomotor activities. His speech was a bit slow and he seemed to exert more effort in understanding questions. Our patient exhibited an appropriate affect and seemed to be in an irritable mood. Delusion, hallucination, and illusion were not found. Thought process was normal, but we noted that his judgment was disturbed. We marked that his insight related to his current condition was 4 and also found his short-term memory was also disturbed. Other laboratory parameters, such as blood check and thyroid, were found to be expected.

Our patient was diagnosed with gaming disorder with detailed multi-axial diagnoses as the following: Axis I: Gaming disorder, Axis II: Anankastic personality, Axis III: None, Axis IV: Environment, AND Axis V: GAF 60-51. We also applied game disorder scoring on to our patient using IGD Scale-Short-Form $($ IGDS9-SF $)=35$.

\section{Discussion}

In rendering patient's diagnosis, it is necessary to render it in accordance with ICD-11 and DSM-5, as shown in Table 1.

Table 1: ICD-11 and DSM 5 criteria for classification of gaming disorder

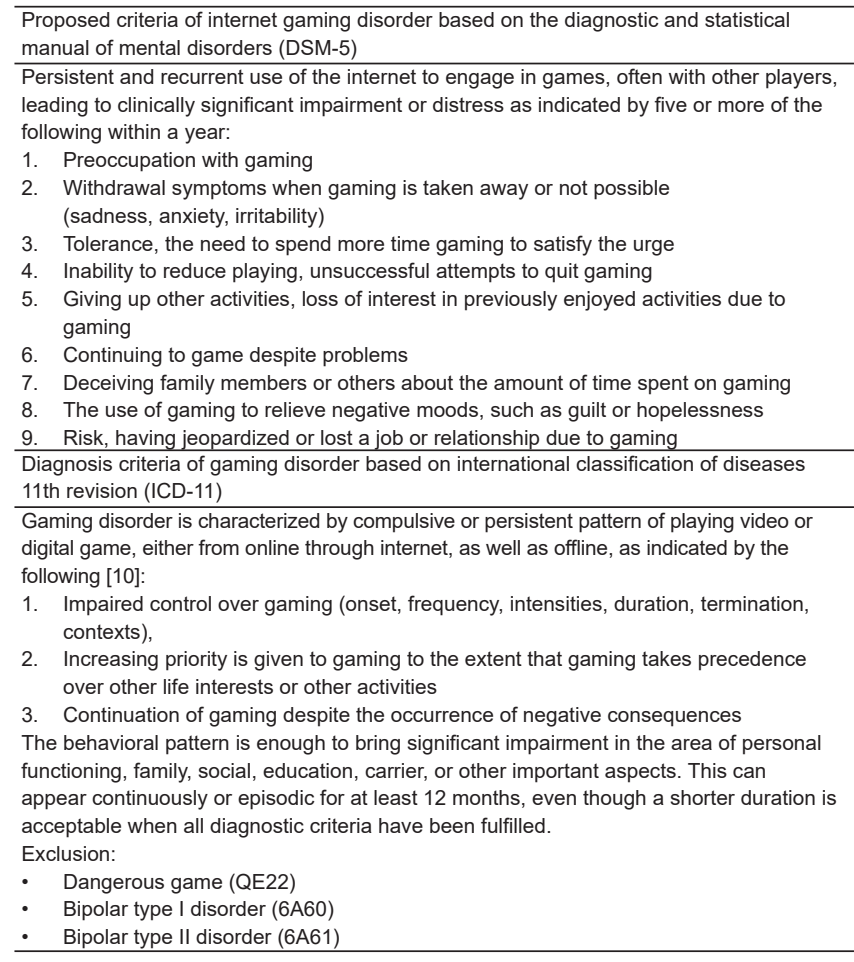


Thus, our patient has met all the diagnostic criteria in which he admitted of playing games for $10-12 \mathrm{~h}$ each day and has been continuously experiencing that for more than 12 months. Our patient was also unable to reduce playing despite several attempts that he has taken. Impairment in his study is greatly marked as our patient puts even more priority in playing game and failed to complete his assignment.

Particularly in our patient's case, his duration of playing game was increased throughout the pandemic from previously $6-7 \mathrm{~h}$ to $10-12 \mathrm{~h}$ a day as he spends even more time staying at home which means even higher needs of entertainment, especially online games. In USA alone, it was reported that there was a significant increase of online gaming for as much as $75 \%$ at the beginning of March 2020 when the USA started to oblige protocols to prevent further spread of Covid-19 [3]. Risk factors that contribute to gaming disorder are the following; biological, personality factor, psychological vulnerability, environmental factor, stress, and structure factors [8]. As for our patient, D was seemed to be lonely and lack of family attention as his parents are busy working.

We suggest that our patient's case can give more vivid and wider picture of gaming disorder, particularly the fact that in D's case, the duration of playing game is greatly increased, which rarely reported in other previous studies. We also point out that environmental factor plays a significant role in the development of gaming disorder.

\section{Conclusion}

Gaming disorder during pandemic still has not been greatly reported, particularly in the presence of increased playing duration, as shown in our patient. We believe that further studies are necessary to completely understand this particular disorder and be able to address it in the future.

\section{References}

1. Elhai JD, Yang H, Mckay D, Asmundson GJ. COVID-19 anxiety symptoms associated with problematic smartphone use severity in Chinese adults. J Affect Disord. 2020;274:576-82. https://doi. org/10.1016/j.jad.2020.05.080

PMid:32663990

2. King DL, Delfabbro PH, Billieux J, Potenza MN. Problematic online gaming and the COVID-19 pandemic. J Behav Addict. 2020;9(2):184-6. https://doi.org/10.1556/2006.2020.00016

PMid:32352927

3. Ko C. Impact of COVID-19 on gaming disorder: Monitoring and prevention. J Behav Addict. 2020;9(2):187-9.

PMid:32634111

4. Amin KP, Griffiths MD, Dsouza DD. Online gaming during the COVID-19 pandemic in India: Strategies for work-life balance. Int J Ment Health Addict. 2020;2020:1-7. https://doi.org/10.1007/ s11469-020-00358-1

PMid:32837441

5. Wang Q, RenH, Long J, LiuY,LiuT.Research progressand debates on gaming disorder. Gen Psychiatry. 2019;32(3):e100071. https://doi.org/10.1136/gpsych-2019-100071

6. Jo YS, Bhang SY, Choi JS, Lee HK, Lee SY, Kweon Y. Clinical characteristics of diagnosis for internet gaming disorder: Comparison of DSM-5 IGD and ICD-11 GD diagnosis. J Clin Med. 2019;8(7):945. https://doi.org/10.3390/jcm8070945

PMid:31261841

7. American Psychiatric Association. Diagnostic and Stastistical Manual of Mental Disorder. Philadelphia, PA: American Psychiatric Association; 2013.

8. Torres-Rodríguez A, Griffiths MD, Carbonell X. The treatment of internet gaming disorder: A brief overview of the PIPATIC program. Int J Ment Health Addict. 2018;16(4):1000-15. https:// doi.org/10.1007/s11469-017-9825-0 PMid:30147635

9. Torres-rodríguez A, Griffiths MD, Carbonell X, Oberst U. Internet gaming disorder in adolescence: Psychological characteristics of a clinical sample. J Behav Addict. 2018;7(3):707-18. https:// doi.org/10.1556/2006.7.2018.75

PMid:30264606

10. Statistics M. International Classification of Diseases for Mortality and Mordibidity Statistics Elevent Revision; 2018. 